\title{
SOCIÉTÉ ET JEUNES COUPLES À TLEMCEN ET SON HAWZ: ENTRE PRESCRIPTIONS ET PROSCRIPTIONS ALIMENTAIRES
}

\author{
Society and Young Couples in Tlemcen and Its Peri-Urban Area \\ Between Alimentary Prescriptions and Proscriptions
}

\author{
Mustapha GuenaOU ${ }^{1}$ \\ CRASC, Orán (Argelia)
}

\section{Résumé}

Tlemcen, ancienne capitale du Maghreb, est connue pour ses spécialités culinaires dont les origines remontent à l'arrivée massive des andalous en Afrique du Nord. Ce travail entre dans le cadre des résultats d'une enquête de terrain dans le Tlemcenois (période allant de 2006 à 2010) : Tlemcen (médina et l'intra muros de Tlemcen) et son hawz (l'extra muros de la médina). Nous avons remarqué quelques particularités et singularités, lors de nos enquêtes portant sur la vie sociale et culturelle de la population locale, tout en insistant sur les rituels de ces citadins et ces semi ruraux. Notre travail étudie l'alimentation recommandée ou interdite pour un jeune couple. L'intérêt de cette contribution touche le domaine de la socio-anthropologie, surtout dans le cas des recommandations et des interdits alimentaires, pendant la période allant depuis la nuit des noces jusqu'à la naissance du premier enfant. A cet effet, nous avons projeté de présenter une étude qualitative et l'approche adoptée serait socio anthropologique pour mieux appréhender les conditions des recommandations et des interdits alimentaires dans une société dite conservatrice et traditionnaliste et surtout identifier les raisons de ce choix, ayant pour source d'inspiration la peur ou la crainte. Dans ce cadre, il est nécessaire de faire appel à l'observation participante - une observation du comportement des parents et des beaux parents en situation relationnelle-, accompagnée d'entretiens, formels et informels, avec les couples de nouveaux mariés. Notre travail vise une réflexion portant sur l'organisation et la portée de cette pratique sociale qui reste

1 Profesor e investigador asociado del Centre de Recherche en Anthropologie Sociale et Culturelle (CRASC-Orán). Miembro y corresponsal del Centre National des Études Historiques (CNEH) de Argel. Correo electrónico : guenaoum@yahoo.fr. Fecha de recepción del artículo : 30 de octubre de 2015 . Fecha de aceptación y versión final : 10 de enero de 2016

STVDIVM. Revista de Humanidades, 22 (2016) ISSN: 1137-8417, pp. 209-223 
encore d'actualité dans une médina, objet de mon champ d'action et de recherche. Notre problématique se limite à une interpellation de la mémoire collective locale : pourquoi ces recommandations et ces interdits alimentaires sont-ils respectés, uniquement, par les jeunes couples?

Mots-clés : jeunes couples, Tlemcen, hawz, alimentation, interdits alimentaires

\section{Resumen}

Tlemcen, la antigua capital del norte de África, es conocida por sus especialidades culinarias cuyos orígenes se remontan a la llegada masiva de andalusíes al norte de África. Este trabajo es parte de los resultados de un estudio de campo en la región de Tlemcenois: Tlemcen (la medina e intramuros de Tlemcen) y su hawz (los arrabales o extramuros de la medina). En nuestras investigaciones sobre la vida social y cultural de la población local, registramos algunas singularidades y particularidades en torno a los rituales de sus ciudadanos y de los habitantes del entorno periurbano y semirural. Nuestro estudio se centra en el análisis de la dieta recomendada y la dieta prohibida para las parejas de recién casados, sobre todo de las prescripciones y restricciones dietéticas durante el período transcurrido desde la noche de bodas hasta el nacimiento del primer hijo. Para ello, se ha llevado a cabo un estudio cualitativo, con un enfoque antropológico social, que ha posibilitado comprender mejor los condicionantes de estas recomendaciones y restricciones en la dieta de una sociedad tradicionalista y conservadora y, sobre todo, identificar las razones de una elección alimentaria basada en el miedo o en el temor. La metodología aplicada está basada en la observación participante (observación del comportamiento de los padres y futuros padres en situación relacional) acompañada de la realización de entrevistas formales e informales a las parejas de recién casados. El artículo ofrece una reflexión sobre la organización y el alcance de esta práctica social — todavía relevante en la medina objeto de nuestra investigación- y una interpelación a la memoria colectiva local: ¿por qué estas recomendaciones y restricciones dietéticas son respetadas únicamente por las parejas jóvenes?

Palabras clave: parejas jóvenes, Tlemcen, hawz, alimentación, normas dietéticas

\section{INTRODUCTION}

En premier lieu, l'idée reçue de nos aînés est encore d'usage dans le monde auquel nous appartenons : il s'agit de celle qui nous rappelle que le corps humain vit harmonieusement avec deux consommations. La première lui donne la vitalité et les efforts physiques et l'autre est celle qui donne l'occasion au corps de communiquer et de vivre son harmonie intérieure que nous associons à la vie du couple. 
Pour cette raison, la sexualité est considérée comme naturelle et une importante activité, source de compréhension et de communication entre les deux partenaires. Leurs difficultés peuvent être facilement évitables par un consentement et une forme de cohabitation, basée sur les principes de l'écoute et de l'échange mutuels.

En second lieu, la religion est liée à une doctrine et une croyance mais les pratiques sociales demeurent étroitement liées au comportement et à la culture des individus dans un même espace socio culturel. La religion mahometane ne demande pas mais exige aux pratiquants de se conformer aux prescriptions et proscriptions dictées dans le cadre de leur vie sociale, culturelle et cultuelle. On ne s'intéresse pas aux prescriptions et aux proscriptions alimentaires en Islam. On n'ose pas parler, dans cette contribution, des aliments licites et les aliments illicites dans la religion musulmane.

Plusieurs religions prescrivent des interdits qui se lient directement aux tabous alimentaires. Quant à nous, nous parlerons des interdits culturels (tabous socioculturels) et des interdits cultuels (interdits socio-cultuels).

Notre contribution ne s'intéresse pas à la religion et ses interdits alimentaires, ni au halal (licite à la consommation) et au haram (illicite à la consommation), chez telle ou telle religion; mais nous allons toucher de près la tradition d'une population, ayant acquis une culture nouvelle toute en abandonnant sa ruralité. Cette culture nous renvoie, principalement, à l'héritage andalou qui nous plonge dans l'art culinaire amélioré.

Il s'agit en effet de pratiques populaires dans la région que nous étudions. Nous associons cette tradition aux pratiques coutumières locales. La vie du corps est fonction de sa vitalité et que son harmonie soit par une alimentation recommandée ou interdite ou par un sentiment de rapprochement corporel. Celui-ci fait de la vie du couple un espace d'échange sentimental et de pérennité.

\section{Premiers constats de notre enquête de TerRain}

Il nous a été donné de constater, lors de nos enquêtes de terrain préliminaires, quelques questionnements qui nous interpellent toujours : il s'agit de l'inventaire des raisons et des causes de ces troubles ou difficultés temporaires ou durables de la sexualité, remarquées ou observées depuis 
les quelques jours précédant la nuit des noces jusqu'à la naissance du premier nouveau-né. Il s'agit des difficultés sexuelles. ${ }^{2}$ Alors, nous sommes arrivés à les répertorier et les classer comme suit :

a) Troubles et difficultés sexuels

- L’impuissance sexuelle définitive.

— Le dysfonctionnement érectile.

b) L'éjaculation précoce

- L'éjaculation prématurée.

- L'éjaculation rapide.

c) L'absence de l'envie et le désir sexuels

- L'absence totale de l'envie de la consommation sexuelle.

- L'absence totale du désir de la consommation sexuelle.

d) Le manque de l'envie et le désir sexuels

- Le manque de l'envie de la consommation sexuelle.

- Le manque du désir de la consommation sexuelle.

e) L'insuffisance de l'envie et le désir sexuels

- L'insuffisance de l'envie de la consommation sexuelle.

- L'insuffisance du désir de la consommation sexuelle. ${ }^{3}$

2 http://sante.lefigaro.fr/mieux-etre/sexualite

3 «Il existe plusieurs classifications des difficultés sexuelles. Les deux principales étant celle de l'Organisation mondiale de la santé (ICD-10) et celle de l'Association psychiatrique américaine (le DSM-IV-TR qui sera bientôt remplacée par le DSM-V). Dans les grandes enquêtes sur la sexualité, chaque pays choisit en outre son propre système de questions sur la sexualité. Pour les hommes, de façon générale, un consensus existe pour considérer surtout les problèmes suivants : les difficultés d'érection (= "dysfonction érectile », autrefois appelée "impuissance ») ; l'éjaculation précoce (= éjaculation prématurée = éjaculation rapide) ; le manque ou l'absence de désir sexuel. Certaines grandes enquêtes recensent cependant d'autres difficultés : absence d'orgasme, sexe sans plaisir, douleurs lors du rapport sexuel, anxiété à propos de la performance (exemples tirés des enquêtes nationales américaine et australiennes). Enfin, les sexologues sont encore plus larges puisqu'ils prennent en charge toutes les difficultés liées à la sexualité, à partir du moment où une souffrance s'exprime : addiction sexuelle, perversion sexuelle (= paraphilie), identité sexuelle, troubles de l'image du corps, etc. Les difficultés sexuelles sont relativement fréquentes. Dans la dernière grande enquête nationale sur la sexualité des français, 9\% des hommes déclarent être "souvent » confrontés à au moins l'une des difficultés sexuelles suivantes au cours des 12 mois écoulés : difficulté d'érection (2,5\%), éjaculation précoce $(6,5 \%)$, absence ou insuffisance de désir (1,9\%). Ils sont encore plus nombreux à l'être 
Ce questionnement nous oriente vers la problématique que nous allons traiter dans cette contribution. A cet effet, notre travail se limite, principalement, à l'interpellation de la mémoire locale pour pouvoir aborder le sujet de prescriptions et de proscriptions alimentaires pendant une période qui nous renvoie à quelques jours de la nuit des noces du couple jusqu'à la naissance de leur premier enfant. Sachant bien, comme le démontrent les résultats de la recherche scientifique, que la consommation sexuelle a pour origine un «quelque chose ", étroitement lié au comportement, à la pensée, au psychique, à l'émotion et au sentiment de l'un par rapport à l'autre. Parfois, l'homme doute de sa masculinité et sa virilité devant une femme réceptive par sa féminité qui, normalement pensée, est féconde. Devant cette situation qui implique les partenaires individuellement ou collectivement, nous avons orienté notre sujet vers les prescriptions et les proscriptions alimentaires afin de mettre en avant les principes d'une culture locale, loin des recommandations cultuelles.

\section{LES PRESCRIPTIONS ALIMENTAIRES}

\subsection{La prescription de la djarwza daqqa et autres mets pour la femme}

Par définition, la djawza eddaqa (littéralement, la petite noix) est un grain qui ressemble à celui du poivre noir mais de couleur qui tend vers le marron (Fatima Zohra M.). En petite quantité, elle doit être présentée sous deux formes : a) liquide, obtenu à partir d'une opération de macération, est pris avec de l'eau ou du lait ; b) grains moulus et mélangés à une soupe. Pour ces variantes, sa consommation, réservée uniquement aux femmes, est quotidienne et que la prise se fait peu de temps avant la consommation sexuelle.

\subsection{La prescription de ftûr ennafsa}

Pour les femmes en couche, il est recommandé d'offrir un repas exceptionnel, communément appelé ftûr ennafsa, dans le sens d'un repas spécial à la femme en couche (Fatima Zohra M.). Sur la base de notre enquête de terrain, nous sommes arrivés à dénombrer sept variantes de plats réservés à cette catégorie de femmes (Fatima Zohra M.) :

«parfois » : difficulté d'érection $(14,3 \%)$, éjaculation précoce $(33,1 \%)$, absence ou insuffisance de désir (20,2\%). » In : http://sante.lefigaro.fr/mieux-etre/sexualite.

STVDIVM. Revista de Humanidades, 22 (2016) ISSN: 1137-8417, pp. 209-223 


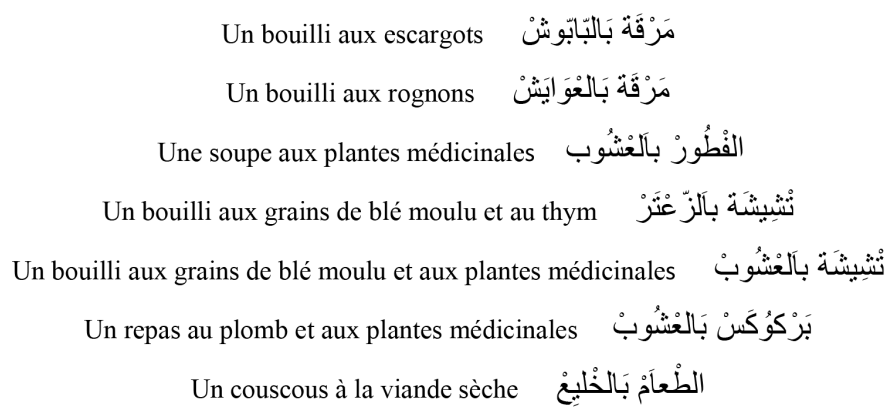

\subsection{Les prescriptions des viandes}

Quelques jours avant les noces, le nouveau marié se lance, sur recommandation des individus de son cercle rapproché, à consommer la ' $a q d a^{4}$ à base de fruits secs et de miel d'abeille. Puis, pendant le repas, sous la surveillance du w-zir (représentation symbolique, lors des noces : ministre chargé des services de l'époux), il peut manger la partie du cou de l'ovin ou du bovin. Cette prescription est renouvelée pour le père, lors de la célébration du septième jour de la naissance du nouveau né.

Par ailleurs, le couple pourra, en commun accord avec les ministres respectifs (même représentation symbolique), manger un repas spécial, communément appelé $M$-hammar (plat à base de viande cuite) ou méchoui (plat à base de viande rôtie). (Hadja Khira B.)

\subsubsection{La composition de la 'aqda pour l'bomme}

Composé d'un mélange à base de noix, d'amandes, des cacahouètes, de pistache et de miel d'abeille, cette 'aqda est prise uniquement par les hommes, surtout les nouveaux mariés. D'ailleurs, elle est recommandable à quelques jours de la nuit des noces.

L'expérience nous a démontré que ce mélange de fruits secs au miel diminue ou réduit, d'une part, les troubles et les insuffisances sexuelles et, d'autre part, l'augmentation du désir charnel. Il s'agit pour certains cas d'une réhabilitation des valeurs de la nutrition traditionnelle (Hadja Khira B.). A titre d'exemple, les pistaches et les noix :

4 Voir infra. 
C'est une réhabilitation totale des valeurs nutritionnelles des pistaches que vient de réaliser une étude menée par un groupe de médecins turcs de l'hôpital Atatürk d'Ankara, a rapporté le Journal international de médecine. Les pistaches, loin d'être un des nombreux aliments indésirables et responsables de la malbouffe, sont au contraire riches en protéines et en fibres et bénéfiques à dose raisonnable pour le système cardiovasculaire. Pour ne rien gâcher, les pistaches profitent aussi aux hommes souffrant de troubles de l'érection. (Vanlerberghe, 2012)

Il y a, par ailleurs, une performance sexuelle remarquée, observée et ressentie auprès d'un grand nombre de nos informateurs, et confirmée par quelques unes de nos informatrices. Cette situation nous renvoyait à un rituel festif et à ses rites d'accompagnement : il s'agit du Naïr (Saridj 2001; Bouterfa 2002), un festin familial et annuel, le jour de l'an agricole coïncidant avec le jour de l'an berbère. Celui-ci coïncide avec le 11 janvier de chaque année. Tous les enfants faisaient des réflexions purement innocentes, en voyant le père bénéficier d'une part très importante par rapport à celle des enfants et de la mère. A titre d'exemple, plusieurs fois, un enquêté m'avait confié : « je faisais une remarque à ma mère, lors de la célébration de cette fête, en lui disant : pourquoi mon père reçoit-il plus que nous ? La réponse de la mère était expéditive, en me disant : « mon fils, le jour où tu grandiras et tu deviendras père, tu auras plus que les autres convives. » (Sid'Ahmed B.)

Plusieurs études épidémiologiques ont déjà démontré les effets bénéfiques des pistaches et des noix sur les maladies cardiovasculaires (les gens qui en mangent une à quatre fois par semaine ont un risque de décès par accident cardiaque réduit de $25 \%$ ) (Vanlerberghe, 2012)

D’autres études nous permirent d'apprendre sur les effets de ce mélange de fruits secs, très répandu dans le Maghreb. (Abdelqader S.)

Les chercheurs ont mené une étude prospective simple sur un panel de 17 hommes mariés consultant pour une dysfonction érectile. Le panel a eu à un régime alimentaire normal mais contenant 100 grammes de pistache par jour, et cela pendant trois semaines. Entre le début de l'étude et la fin du régime riche en pistaches, le score moyen de l'index international de la fonction érectile (IIEF) est passé de 36 à 54 points, ce qui est une amélioration très claire, remarquent les chercheurs. Le flux sanguin mesuré dans le pénis par Doppler à ultrasons présente également une amélioration très conséquente, ce qui est à mettre en relation avec les bénéfices cardiovasculaires déjà trouvés pour ce type de régime. (Vanlerberghe, 2012)

D'autres personnes préfèrent s'assurer de ce mélange de fruits secs. Celui-ci rappelle ce que nous retrouvons dans notre marché à Tlemcen, 
ancienne capitale du Maghreb central, comme fruits secs, communément appelé El $m$-khallèt —le mélangé- ou El Qashq-sha —les produits mélangés à base de fruits secs- (Abderahman B.) Les fruits secs recommandés pour cette 'aqda sont :

$$
\begin{aligned}
& \text { les amandes اللُّوزْ } \\
& \text { les noix القُرْقَاَعُ (الجَوْزَ) }
\end{aligned}
$$

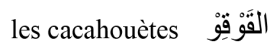

$$
\begin{aligned}
& \text { les graines de sésame rouge } \\
& \text { الَجَنْجَلَنْ الاَحْمَز } \\
& \text { un ensemble d'épices moulues puis mélangées حرَاجْ رَمْضَنَانْ (Karima B.) }
\end{aligned}
$$

Il est nécessaire de rappeler que certaines familles de l'intra muros ou de l'extra muros de Tlemcen préfèrent ajouter d'autres grains à usage médicinal tels que :

$$
\begin{aligned}
& \text { El horf الحُرْفْ (شَوَيَة) } \\
& \text { Zari'ats El kettsan زَرِعَة الكَّنَانْ (sic) (Karima B.) }
\end{aligned}
$$

\subsubsection{Les vertus de la 'aqda et des autres mets}

Ces prescriptions se présentent sous forme de lutte contre l'impuissance physique temporaire ou définitive chez les hommes, surtout en cette période, alors considérée comme cruciale puisqu'elle débute, sept jours avant la célébration du rituel festif familial, communément appelé les noces. À Tlemcen et à Ain El Hûts, représentant respectivement la population citadine et l'intra muros et la population semi rurale et l'extra muros du Tlemcenois, cette pratique est courante et observable. Selon nos informatrices et nos informateurs, ces mets sont alors considérés comme produits de lutte contre toute forme d'impuissance sexuelle, dans l'esprit de la femme comme dans la culture de l'homme. D'ailleurs, il est nécessaire de rappeler que les parents et les familles respectives du couple attendent deux choses importantes dans la vie du couple : la perte de la virginité de la mariée par la virilité de son époux ; et le rapport sexuel fécondateur par la masculinité virile de l'homme et la féminité réceptrice de la femme. (Belkacem M.)

Cette efficacité doit, principalement, entrainer une harmonie idéale de la vie de couple, basée sur les principes de la vie du corps et de sa sexualité, sa sensualité et sa communication avec le partenaire. Cette harmonie de la 
vie du couple est effective pour pouvoir lutter contre les préjugés et la méchanceté gratuite des envieux et des pervers, dans les milieux féminins et masculins.

Le travail du terrain, des années durant, nous permit de comprendre que plusieurs accidents psychologiques sont la cause de nombreuses calamités socioculturelles aux quelles sont confrontées des familles dont la plupart perdent la notion de lien familial.

Dans ce contexte de lutte contre l'impuissance sexuelle, que nous associons aux troubles sexuels temporaires ou durables, nous évoquons l'importance de ces recommandations, liées à la consommation d'un met fortifiant. Celle-ci s'explique par des troubles d'érection chez l'homme ou de baisse de libido dont les sources et les causes sont d'origines diverses. Pendant et après la nuit des noces, cette situation, selon l'esprit et la mentalité de la population locale, est inquiétante pour le couple comme pour leurs parents respectifs.

Pour prévenir ou remédier à cette situation très délicate, la population locale fait appel aux vertus de ces produits, de fabrication locale et traditionnelle. Dans certains cas, cette situation est transitoire et nécessite uniquement la consommation de ces mets pour une période courte; et s'il s'agit d'une situation durable ou persistante, le couple est appelé à aller rendre visite à des spécialistes et à l'usage des médicaments, mis à la disposition de ces malades qui nécessitent un traitement médical et non l'usage de certaines plantes médicinales.

Ces mets, ayant des principes actifs sur la fécondité, ont pour effets d'encourager, en premier lieu, l'attractivité corporelle pour une vie sexuelle normale. Puis, en seconde phase, une préparation psychologique du couple qui veut honorer les parents respectifs par l'annonce d'une mise au monde d'un enfant, surtout avec une préférence pour le garçon. Il s'agit d'une particularité des préférences dans le monde arabo-musulman. Ces mets médicamenteux traditionnels se substituent aux médicaments qui permettent au corps de vivre son harmonie par l'échange mutuel et la communication corporelle.

Cette situation, observée des années, voire des décennies durant, nous permit de comprendre le fonctionnement. Scientifiquement, il s'agit du fonctionnement de ces mets par la double circulation du sang. Ils assurent au couple l'harmonie corporelle, ressentie à tout moment de rapprochement physique qui, nécessairement, influe sur le moral, source d'inspiration et de volonté sexuelle. L'effet ne s'arrête pas ; au contraire, il dure longuement 
par les vertus des produits utilisés dans la composition. Cette dernière vivifie l'envie et assure la lutte contre les effets qui ralentissent la rapidité et le taux d'efficacité du produit consommé, à des moments précis de la journée et de la soirée. (Hadj Abdelkrim N.)

Quelques données scientifiques peuvent apparaittre pour assurer un effet positif sur la vie du couple et sur l'harmonie de leur corps respectif. Dans ce cas, nous avons entamé une enquête ${ }^{5}$ qui nous permit, sur un échantillon de cent (100) personnes, entre sexagénaires et septuagénaires, de relever ce qui suit :

- L'efficacité de l'usage.

- L'esprit de l'expérience.

- Les résultats de l'expérience.

Pour le moment, nous ne pouvons aller plus loin que les résultats obtenus à partir d'une enquête de terrain. Les preuves de relation entre le produit et les effets de la consommation restent liées à cette harmonie corporelle, ayant pour source les effets médicamenteux, expliquée par la double circulation du sang et l'effet psychologique qui serait le plus important par rapport au premier. La preuve est là : nombreux sont encore les jeunes couples qui se rabattent sur ces produits pour faire plaisir à son partenaire et aux parents respectifs.

À titre indicatif, une étude est entamée, depuis quelques mois, sur les raisons de la chute de la libido, la perte temporaire ou durable de la libido et de l'impuissance sexuelle que nous associons à l'appétit sexuel. Cette étude vise, essentiellement, la compréhension de la mentalité de la population locale et l'usage de ces mets dit « magiques » ou « fortifiants».

Il y a plusieurs explications des troubles sexuels, chez l'homme comme chez la femme, lors de la célébration de la nuit des noces. La classification de ces troubles ou difficultés temporaires ou durables nous renvoient à une problématique, liée au régime alimentaire ou à l'état de santé des individus qu'il soit physique ou psychique. (Hadj Abdelkrim N.)

5 Quelques données statistiques:

\begin{tabular}{|lccc|}
\hline Effets & Hommes & Femmes & Totanx \\
\hline Effets positifs & $40 \%$ & $42 \%$ & $82 \%$ \\
Effets négatifs & $02 \%$ & $01 \%$ & $03 \%$ \\
Sans réponses & $08 \%$ & $07 \%$ & $15 \%$ \\
Totaux & $50 \%$ & $50 \%$ & $100 \%$ \\
\hline
\end{tabular}




\section{LES PROSCRIPTIONS ALIMENTAIRES}

Dans le cadre des proscriptions, nous avons axé notre intervention sur les proscriptions alimentaires de la nuit des noces, pendant la grossesse et les quarante jours de la naissance du nouveau-né.

\subsection{Les noces}

Depuis l'annonce et de la fixation de la date des noces, le couple est censé être averti par leurs proches et leurs préposés respectifs des deux époux afin d'éviter :

a) de manger de la main d'un étranger ou d'une étrangère au cercle rapproché du couple.

b) de prendre ou de consommer une boisson d'un étranger ou d'une étrangère au cercle rapproché du couple.

c) d'accepter toute forme d'invitation d'une personne étrangère au même cercle rapproché du couple. (Abdellah B.).

Cette recommandation est assimilée à une proscription alimentaire concernant uniquement le couple. Les mêmes proscriptions doivent être respectées pendant toute la période du déroulement du rituel festif familial ou les noces.

Pendant cette période, le cercle rapproché du couple se réduit au maximum à une ou deux personnes à savoir la mère ou la sœur aînée et les w-zirs (les chargés des services du marié et celle réservée à la mariée).

Le couple est privé de consommer, pendant la période des noces,

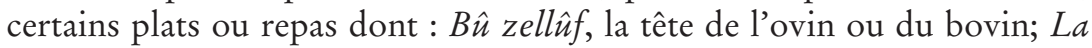
dîawara, les abats de l'ovin ou du bovin; et El-lssène, la langue de l'ovin ou du bovin.

\subsection{La grossesse}

La femme enceinte est privée de la consommation des variantes de plats à base de Djawza Eddaqa.

\subsection{La période de couche}

Il est formellement interdit à toute femme en couche de consommer :

- La Djarza eddaqa, alors recommandée uniquement aux femmes aptes ou dans le besoin de faciliter l'ovulation. 
- Bûz zellûf (la tête de l'ovin ou du bovin).

- La dûawara (les abats de l'ovin ou du bovin).

- El-ssène (la langue de l'ovin ou du bovin).

- Le foie de l'ovin et du bovin.

Ces interdictions ont pour origine uniquement la superstition, très répandue dans la société étudiée. Par ailleurs, certaines familles de Tlemcen et d'Ain El Hûts interdisent à leurs brus, en période d'envie, de manger certains plats à base de :

- Escargot jaune.

- Esophage.

- La viande du lapin.

- Bîu zellûf (cf. supra).

- La dûawara (cf. supra).

- El-ssène (cf. supra).

\section{ConClusion : RAISONS ET EXPLICATIONS DES PRESCRIPTIONS ET DES PROSCRIPTIONS}

Sur la base de l'enquête de terrain, nos informatrices et nos informateurs, lors de nos différents entretiens, nous permirent de relever :

a) Les signes de la superstition en usage dans la société (pessimisme).

b) Les signes de présage et de vœux (optimisme).

c) Les évitements de certaines conséquences physiologiques des nouveau-nés.

Si les difficultés sont évitables, ça ne permet pas de dire que la vie du couple est parfaite, bien qu'elle soit idéale. Devant la normalité de la vie harmonieuse du corps et de sa performance, le couple se trouve affronté à quelques difficultés, d'ordre comportemental et sentimental ; mais il s'agit des exigences qui s'expliquent par la communication lors d'un rapprochement corporel que vénère les partenaires d'une vie sexuellement commune.

Nos travaux d'enquête se poursuivent dans de bonnes conditions. Quant aux enquêtes de terrain, elles nous permettront de vérifier d'autres hypothèses telles que la frigidité, le manque de l'orgasme, l'absence total de l'orgasme, la performance sexuelle, l'anxiété, le stress, etc. 
Ces mêmes enquêtes nous aideront à mieux comprendre les phénomènes, liés à la sexualité dont la dépendance sexuelle, la perversion sexuelle ou paraphilie, les troubles de l'image du corps de l'un par rapport à celui de son partenaire.

La conception de la consommation sexuelle ne se limite pas uniquement à une affaire d'usage mais il faut lui assurer une dimension et une présentation de l'image. Pour conclure : Que se passe-t-il dans ce monde ?

\section{RÉFÉRENCES}

AвоU Bekr, Abdeslam, 1936, «Usage de droit coutumier dans la région de Tlemcen », Revue Africaine, 79/2 : 813-866.

AвоU BeKR, Abdeslam, 1937 « Notes sur les amulettes chez les indigènes algériens ", Revue Africaine, 81 : 309-318.

BeAussier, Marcelin, 1958, Dictionnaire Arabe-Français. Nouvelle édition, revue, corrigée et augmentée par Mohammed Bencheneb. Alger, La maison des livres. 2 tomes

Benachenhou, Abdelhamid, 1971, Connaissance $d u$ Maghreb. Notions d'éthographie, d'bistoire et de sociologie. Alger, Editions Populaires de l'Armée.

Boubekeur, Cheikh Si Hamza, 1990, Un soufi algérien Sidi Cheikh. Sa vie, son œuvre, son rôle historique, ses descendants (Oulâd Sidi-Cheikh). Paris, Maisonneuve \& Larose.

Boucherit, Aziza, 2006, L'arabe parlé à Alger. Alger, Editions ANEP.

BOUDJEDRA, Rachid, 1971, Vies quotidiennes Contemporaines en Algérie. Paris, Hachette.

BouhadibA, Abdelwahab, 1982, La sexualité en Islam. Paris, Quadrigue-PUF.

Bouterfa, Said, 2002, Yennayer ou le symbolisme de Janus. Alger, Musk Editions.

Cazeneuve, Jean, 1971, Sociologie du rite. Paris, PUF.

Coran, Le. Traduction nouvelle par le Cheikh Boubakeur Hamza. Alger, ENAG éditions, 1989. 2 tomes.

Delteil, Joseph, 1976, Le sacré corps. Paris, Grasset.

EL KENZ, Ali, 2005, «Les sciences humaines et sociales (SHS) dans les pays arabes de la Méditerranée », Insaniyat, 27 (Janvier-Mars 2005) : 19-28.

El Medjdoub, Abderrahman, Le derwiche, [2001], Adages et maximes. Textes recueillis par Rouaï Amina. Préface de Haldaoui Mamoune. Oran, Dar El Gharb.

Elimam, Abdou, 2003, Le Maghribi alias "ed-darija». La langue consensuelle $d u$ Maghreb. Oran, Dar El Gharb.

Gaudefroy-Demombynes, M., 1901, Les cérémonies du mariage chez les indigènes de l'Algérie. Paris, Maisonneuve.

Halbwachs, Maurice, 1968, La mémoire collective. Paris, PUF. 
MAHDJOuB, Abderrahmane, 1954, «Fêtes et coutumes musulmanes », Richesses de France, $18:$ (n. ${ }^{\circ}$ spécial: Tlemcen et sa région) : 47-52.

MAhmoud, Yahia, 1986, La thérapeutique par les plantes les plus communes en Algérie. Blida, Le Palais du Livre.

Maunier, René, 1935, Coutumes algériennes. Paris, Domat-Montchrestien.

NousCHI, A., 1986, « La consommation des glands en Kabylie. » In Encyclopédie Berbère. Alger-Amzwar, Peeters. Tome IV : 487. (https://encyclopedieberbere. revues.org/2436\#tocto1n3)

Nouvel, J., 1939, «L'enfance musulmane indigente à Rabat. » In Quatrième congrès de la Fédération des Sociétés Savantes de l'Afrique du Nord Rabat 1820 avril 1938. Alger, Société Historique Algérienne: 737-761.

PlatTI, Emilio, 1994, "Le sacrifice en Islam. » In Le sacrifice dans les religions, sous la direction de Marcel Neusch. Paris, Beauchesne: 157-174.

RACHIK, Hassan, 1990, Sacré et sacrifice dans le haut Atlas marocain. Casablanca, Afrique Orient.

Rahmani, Slimane, 1935, "Le mois de mai chez les Kabyles », Revue Africaine, $76: 361-366$.

Rahmani, Slimane, 1936, "Rites relatifs à la vache et au lait ", Revue Africaine, 79/2: 791-809.

Rahmani, Slimane, 1937, "La grossesse et la naissance au Cap Aokas ", Revue Africaine, $81: 217-245$.

RAHMANI, Slimane, 1939, "L'enfant chez les Kabyles jusqu'à la circoncision. » In Quatrième congrès de la Fédération des Sociétés Savantes de l'Afrique du Nord Rabat 18-20 avril 1938. Alger, Société Historique Algérienne. Vol. II : 815-842.

Rahmani, Slimane, 1939, "Coutumes kabyles du Cap Aokas. Deuxième partie. L'enfance de la naissance à la circoncision », Revue Africaine, 83 : 65-120.

SARIDJ, Mohamed, 2001, Verveine Fanée. Coutumes et traditions du pays des BeniSnous. Oran, Dar El Gharb.

Scelles-Millie, Jeanne, 1979, Traditions algériennes. Paris, Maisonneuve et Larose.

SHINAR, Pessah, 1983, Islam maghrébin contemporain. Bibliographie annotée. Paris, Editions du CNRS.

Sironneau, Jean-Pierre, Métamorphoses du mythe et de la croyance. Paris, L'Harmattan.

Temple, Dominique et Mireille Chabal, 1995, La réciprocité et la naissance des valeurs humaines. Paris, L'Harmattan.

WARING, Philippe, 1982, Dictionnaire des présages et des superstitions. Traduit et adapté par Christel Rollinat. Paris, du Rocher.

Westermarck, Edouard, 1922, "Les cérémonies du mariage au Maroc », Revue Algérienne,1922 : 511. 
Zenagui, Abdelaziz 1904, «Récit en dialecte tlemcenien. Préface, traduction et notes par M. Gaudefroy-Demombyne », Journal Asiatique, IV (Juillet-Aout 1904) : 45-117.

Zerdoumi, Nafissa, 1982, Enfants d'bier. L'éducation de l'enfant en milieu traditionnel algérien. Préface de Maxime Rodinson. Paris, François Maspero.

\section{Webographie}

FrEOUR, Pauline, 2012, "Impuissance : un traitement anti calvitie soupçonne. » http://sante.lefigaro.fr/actualite/2012/03/12/17717

VANLerberghe, Cyrille, 2012, « Les hommes qui mangent des pistaches sont meilleurs au lit. » http://sante.lefigaro.fr/actualite/2012/01/13/16928 